\title{
Electromagnetic Noise Source Approximation for Finite-Difference Time-Domain Modeling Using Near-Field Scanning and Particle Swarm Optimization
}

\author{
Ian Scriven, Member, IEEE, Junwei Lu, Senior Member, IEEE, and Andrew Lewis
}

\begin{abstract}
This paper presents an electromagnetic noise source approximation method based on a 2-D array of electric dipoles for use in finite-difference time-domain simulations. The currents (both magnitude and phase) of these dipoles are optimized via a particle swarm algorithm so as to minimize the difference between the magnetic near-field produced by the dipole array and the magnetic near-field produced by the device under test. The method presented requires only the magnitude of the magnetic field to be measured, simplifying the measurement process. The new noise source modeling method has been applied to a transmission-line test case, demonstrating the performance and accuracy of the method.
\end{abstract}

Index Terms-Finite-difference time-domain (FDTD) method, near-field scanning, noise source modeling, particle swarm optimization (PSO).

\section{INTRODUCTION}

$\mathbf{M}$ EETING electromagnetic emissions standards for new electronic products can be an expensive exercise when following the traditional route of physical prototyping and testing, especially if a large number of tests and design revisions are required to achieve compliance. Computational electromagnetics (CEMs) simulation could therefore prove to be a valuable electromagnetic (EM) compatibility (EMC) design tool if it can be utilized during the design process to ensure first-pass compliance with emissions standards. In order to realize such gains in design-process efficiency, however, accurate approximate models for EM noise sources are required, as it is often very difficult or impossible to directly model sophisticated electronic components in CEM simulations.

There are a number of possible methods for modeling the sources of EM emissions from electronic devices. Simplified sources such as a single electric and magnetic dipole antenna, patch antennas, or multiple randomly placed dipoles, currently dominate the field [1]-[5], due to the apparent difficulty in obtaining more accurate (and complex) models. Alternatively, a specially configured array of magnetic or electric dipole

Manuscript received February 18, 2009; revised May 28, 2009. First published January 22, 2010; current version published February 18, 2010. This work was supported in part by the Australian Research Council under Project "A Virtual Electromagnetic Compatibility (EMC) Lab Based on Advanced Computer Modelling and Simulation Techniques."

I. Scriven and J. Lu are with the School of Engineering, Griffith University, Brisbane, Qld. 4111, Australia (e-mail: ian.scriven@ student.griffith.edu.au; j.lu@griffith.edu.au).

A. Lewis is with the Institute for Integrated and Intelligent Systems, Griffith University, Brisbane, Qld. 4111, Australia (e-mail: a.lewis@ griffith. edu.au).

Digital Object Identifier 10.1109/TEMC.2009.2036251 antennas can be used to more accurately represent EM noise sources [6], [7]. This method involves taking near-field measurements of the fields around a physical realization of the device in question, then replicating that near-field as accurately as possible using a collection of dipoles. This can be done using either a direct solution of the equations governing radiation from electric or magnetic dipoles [6], or through computational optimization [7], although the latter process tends to take significantly longer to achieve the required accuracy.

The finite-difference time-domain (FDTD) method is a procedure for solving the differential time-dependent form of Maxwell's equations by approximating the continuous partial derivatives at finite intervals. It is a powerful tool for EMC analysis as it allows a wide range of frequencies to be investigated in a single simulation. One drawback, however, is that the model being simulated must be discretized onto a lattice of cubic or rectangular Yee cells [8], meaning that if an array of dipoles is used to approximate the radiated fields from a given device, those dipoles can only be oriented along the $x$-, $y$ - or $z$-axes. This is an issue for the method described in [6], which directly calculates the required dipole currents, as it requires flexible dipole orientation.

This paper attempts to overcome this obstacle by fixing the orientation of each dipole in the array along either the $x$ - or $y$-axis. Modifying the existing method in this manner is demonstrated to enable the $x$ - and $y$-components of the magnetic nearfield to be modeled very accurately, while introducing significant error in the $z$-component. A particle swarm optimization (PSO) algorithm is then applied to this initial solution in order to reduce this error.

\section{ApPROXIMATE SOURCE MODEL}

Any source model used in an FDTD simulation must meet a number of requirements. Obviously, it must be a time-domain source, and it must adequately cover the range of frequencies that are to be examined. When simulating EM emissions from a device, the frequency range to be examined is typically between $30 \mathrm{MHz}$ and $1 \mathrm{GHz}$, the range specified in the FCC standard for radiated emissions. In the time domain, this range can be achieved using a Fourier series of sinusoids, whose frequencies are selected in order to adequately cover the required spectrum.

In this paper, a 2-D array of electric dipoles is used to approximate the emissions from any arbitrary device. A grid of electric dipoles is used as it is much simpler to model than the complex electronics in modern devices, while being able to 


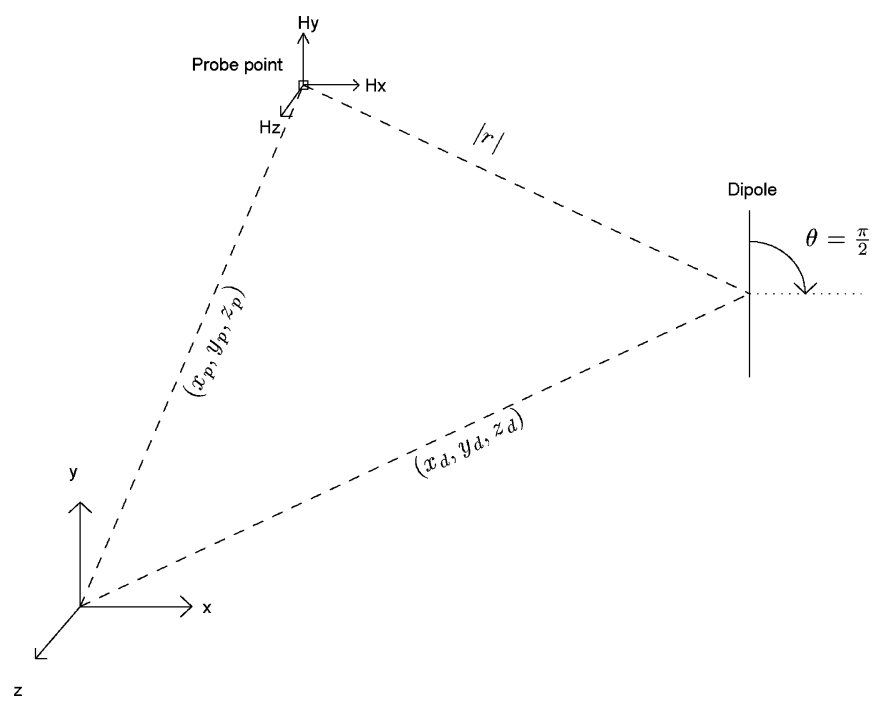

Fig. 1. Magnetic fields produced by an electric dipole in Cartesian coordinates.

reproduce a measured near field with good accuracy, provided enough dipoles are used. This dipole array is oriented in the $x-y$ plane of a 3-D Cartesian grid. In FDTD simulations, the orientation of these dipoles is restricted by this grid, meaning that each dipole must be oriented in either the $x$-direction or the $y$-direction.

The magnetic near-field radiation of these dipoles can be obtained for a given frequency by calculating the curl of the magnetic vector potential. In Cartesian coordinates, the $x-, y$ - and $z$-components of the radiated magnetic field at point $\left(x_{p}, y_{p}, z_{p}\right)$ from an electric dipole of length $\Delta \lambda$ at $\left(x_{d}, y_{d}, z_{d}\right)$ carrying current $I$ at frequency $\omega$ are given in (1), (2), and (3), respectively [6]. The current $I$ is a complex phasor representing both the magnitude and phase of the current, and angle $\theta$ represents the angle subtended with the $x$-axis, as shown in Fig. 1. Using this method, the dipole currents (magnitude and phase) must be calculated at a large number of frequencies in order to build up an accurate, broad-band time-domain model of the EM noise source

$$
\begin{aligned}
& H_{x}= \frac{I}{4 \pi} \Delta \lambda \frac{e^{-j k r}}{r^{2}}\left(\frac{z_{p}-z_{d}}{r}+\frac{j \omega\left(z_{p}-z_{d}\right)}{c}\right) \sin \theta \\
& H_{y}=\frac{-I}{4 \pi} \Delta \lambda \frac{e^{-j k r}}{r^{2}}\left(\frac{z_{p}-z_{d}}{r}+\frac{j \omega\left(z_{p}-z_{d}\right)}{c}\right) \cos \theta \\
& H_{z}=\frac{I}{4 \pi} \Delta \lambda \frac{e^{-j k r}}{r^{2}}\left[-\left(\frac{x_{p}-x_{d}}{r}+\frac{j \omega\left(x_{p}-x_{d}\right)}{c}\right) \sin \theta\right. \\
&\left.+\left(\frac{y_{p}-y_{d}}{r}+\frac{j \omega\left(y_{p}-y_{d}\right)}{c}\right) \cos \theta\right] .
\end{aligned}
$$

Alternatively, the approximate dipole currents can be obtained in the time domain using the Biot-Savart law, which states that the contribution to the total magnetic field at a point from a segment of wire carrying current $I$ is

$$
\Delta \mathbf{B}=\frac{\mu_{0}}{4 \pi} \frac{I \Delta \lambda \times \hat{\mathbf{r}}}{r^{2}}
$$

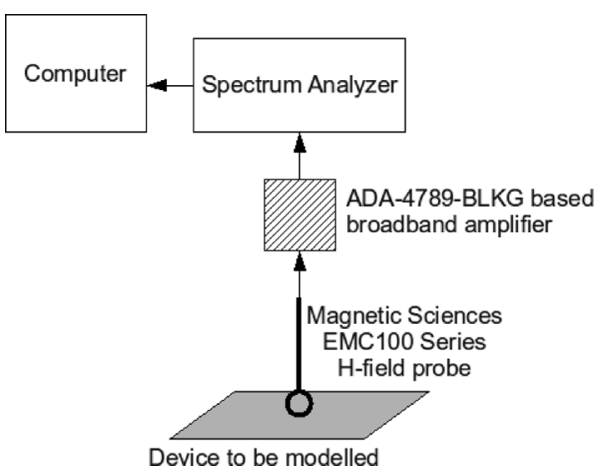

Fig. 2. Proposed magnetic field measurement apparatus.

where $\hat{\mathbf{r}}$ is a unit vector directed from the wire segment toward the measurement point. The Biot-Savart law applies to static fields; however, it provides an accurate approximate to timevarying fields when $r \ll \lambda$.

As time-domain measurement of magnetic fields is very difficult at high frequencies, it is much more convenient to use frequency-domain measurements when calculating the dipole currents required to match the true near-field of the device under test. However, given the relative simplicity of calculating the magnetic field produced by an array of dipoles carrying known currents in the time domain, optimization algorithms can be applied to search for the most suitable dipole currents.

In order to solve this so-called inverse problem, the accuracy of each potential solution must be calculated compared to the frequency-domain data obtained from testing of the device to be modeled. As such, a Fourier transform is required, so the magnetic fields produced by the dipole model must be calculated over a large number of time steps. This method was found to be significantly slower than the previously discussed frequencydomain method, even though such a method must be evaluated over a large number of frequencies to adequately cover the required spectrum.

\section{Calculation of Dipole CuRrents}

\section{A. Acquiring Near-Field Data}

Before an approximate source model can be constructed, magnetic field measurements must be performed over a grid of points in the near-field of the device to be modeled. The height above the device at which the measurements are performed is not overly important, as long as all measurement points are located well within the near-field region. The proposed measurement configuration can be seen in Fig. 2. The magnetic field probe is positioned over the device to be modeled at each measurement point, oriented in such a way to pick up only a single component of the magnetic field. The Magnetic Sciences EMC-100 series magnetic field probes respond only to fields orthogonal to the pickup loop, and are electrostatically shielded to provide isolation from common-mode signals. The position and orientation of the probe can be controlled either manually or with the assistance of a robotic arm [6]. The current induced in the probe is amplified and measured on a spectrum analyzer, and the measured magnetic field is calculated using the probes' calibration data. 


\section{B. Seeding the Optimizer}

This second step in source-modeling process presented in this paper is to calculate a seed solution. It is so named as it identifies the region in the solution space around which accurate approximate models are likely to be found. The term solution space is used here to mean the many-dimensional hypervolume containing all possible combinations of current phasors used in the dipole array approximate model.

The seed solution is computed using a direct solution of (1)(3). As mentioned previously, all dipoles are constrained to be oriented directly along either the $x$ - or $y$-axis. Therefore, for the $x$-direction dipoles (i.e., the dipoles that can be defined as a line between two endpoints $\left(x_{1}, \hat{y}, \hat{z}\right)$ and $\left(x_{2}, \hat{y}, \hat{z}\right)$, where $\hat{y}$ and $\hat{z}$ are constants), (1)-(3) can be rewritten as follows:

$$
\begin{aligned}
H_{x} & =0 \\
H_{y} & =\frac{-I_{x}}{4 \pi} \Delta \lambda \frac{e^{-j k r}}{r^{2}}\left(\frac{z_{p}-z_{d}}{r}+\frac{j \omega\left(z_{p}-z_{d}\right)}{c}\right) \\
& =I_{x} \alpha_{x} \\
H_{z} & =\frac{I_{x}}{4 \pi} \Delta \lambda \frac{e^{-j k r}}{r^{2}}\left(\frac{y_{p}-y_{d}}{r}+\frac{j \omega\left(y_{p}-y_{d}\right)}{c}\right) .
\end{aligned}
$$

Likewise, for all the $y$-direction dipoles, the same magnetic field equations can be rewritten as follows:

$$
\begin{aligned}
H_{x} & =\frac{I_{y}}{4 \pi} \Delta \lambda \frac{e^{-j k r}}{r^{2}}\left(\frac{z_{p}-z_{d}}{r}+\frac{j \omega\left(z_{p}-z_{d}\right)}{c}\right) \\
& =I_{y} \alpha_{y} \\
H_{y} & =0 \\
H_{z} & =\frac{-I_{y}}{4 \pi} \Delta \lambda \frac{e^{-j k r}}{r^{2}}\left(\frac{x_{p}-x_{d}}{r}+\frac{j \omega\left(x_{p}-x_{d}\right)}{c}\right) .
\end{aligned}
$$

From this, it can be seen that the $y$-component of the magnetic field around the approximate model is only dependent on the currents in the $x$-direction dipoles, and the $x$-component of the magnetic field is similarly dependent on only the currents in the $y$-direction dipoles. Using these equations in conjunction with measured data from the device to be modeled, it is trivial to develop an array of such dipoles that will very accurately model the $x$ - and $y$-components of the device's magnetic near field, using the following equations:

$$
\begin{aligned}
& {\left[I_{x}\right]=\left[\alpha_{y}\right]^{-1}\left[H_{y}\right]} \\
& {\left[I_{y}\right]=\left[\alpha_{x}\right]^{-1}\left[H_{x}\right] .}
\end{aligned}
$$

If $z$-direction dipoles were used, the $x$-component of the magnetic field would no longer be dependent on only the $y$-direction dipoles, and would instead be related to the $z$-direction dipole currents as well. Similarly, the $y$-component of the magnetic field would also be dependant on the $z$-direction dipoles as well as those oriented along the $x$-axis, and a direct solution of (1)(3) would no longer be possible due to this overlap.

Such a model will be significantly less accurate in terms of the $z$-component of the magnetic field, as this component was not utilized in its generation. It is possible, however, to increase the accuracy of the generated model in this respect, by slightly altering the current phasors that excite the dipoles. To put it in terms of computational optimization, by searching the previously defined solution space in the region of this seed solution, approximate models that are more accurate overall may be found. In this paper, a particle swarm algorithm is used to perform this search.

\section{PSO Algorithm}

The PSO algorithm is based on swarm intelligence, an artificial intelligence technique that studies collective behavior in decentralized, self-organized groups [9]. It utilizes a population of potential solutions, or particles, which move around the design space with every iteration. The movement of these particles is governed by following two equations [10]:

$$
\begin{aligned}
v_{i j}(t+1)= & w v_{i j}(t)+c_{1} r_{1 j}(t)\left[y_{i j}(t)-x_{i j}(t)\right] \\
& +c_{2} r_{2 j}(t)\left[\hat{y}_{i j}(t)-x_{i j}(t)\right] \\
x_{i j}(t+1)= & x_{i j}(t)+v_{i j}(t+1) .
\end{aligned}
$$

Here, $v_{i j}(t)$ is the velocity of particle $i$ in dimension $j$ at time $t$ and $x_{i j}(t)$ is the position of particle $i$ in dimension $j$ at time $t$. The velocity of a particle depends on both the best position that particle has found to time $t, y_{i j}(t)$ (the local best solution), and the best solution the entire swarm has found to time $t, \hat{y}_{i j}(t)$ (the global best solution). An inertial component is scaled by constant $w$, and $c_{1}$ and $c_{2}$ are constants used to control the impact of the local and global components in velocity equation (13). For the purposes of this work, $w$ was set to 0.3 , and $c_{1}$ and $c_{2}$ were set to 1.4. The vector $r$ is a vector of random numbers evenly distributed between zero and one generated for each particle at each time step $t$.

The PSO algorithm is an iterative one. For the work presented in this paper, the initial position of each particle is randomized around the previously discussed seed solution. Through the use of this prior knowledge of the solution space, faster convergence can be attained [11], [12]. At every iteration, the particle positions are evaluated, the local and global best solutions are updated and new velocities are calculated using (13). Using these new particle velocities, the position of each particle is updated as in (14). The algorithm is terminated when the improvement in the best solution found over a number of iterations drops below a given threshold, in this case $0.1 \%$ over ten iterations.

\section{Evaluation of Potential Solutions}

Particle positions are evaluated using a fitness function, which quantifies the error present in the approximate source model generated by that dipole array configuration. To measure the error, the average (absolute) difference between the measured value and the approximated value of each component of the magnetic field at each measurement point was calculated. Equation (15) shows the fitness function used by this source modeling method:

$$
f(\mathbf{I})=\frac{1}{3}\left(g_{x}(\mathbf{I})+g_{y}(\mathbf{I})+g_{z}(\mathbf{I})\right)
$$


where

$$
\begin{aligned}
& g_{x}(\mathbf{I})=\frac{1}{N} \sum_{i=1}^{N}\left(\sqrt{\left(H_{x \_ \text {real }}^{i}-H_{x_{\text {_approx }}}^{i}\right)^{2}}\right) \\
& g_{y}(\mathbf{I})=\frac{1}{N} \sum_{i=1}^{N}\left(\sqrt{\left(H_{y \_ \text {real }}^{i}-H_{y \_ \text {approx }}^{i}\right)^{2}}\right) \\
& g_{z}(\mathbf{I})=\frac{1}{N} \sum_{i=1}^{N}\left(\sqrt{\left(H_{z \_ \text {real }}^{i}-H_{z \_ \text {approx }}^{i}\right)^{2}}\right) .
\end{aligned}
$$

In this equation, $\mathbf{I}$ is the vector of current phasors being evaluated. Vectors $\mathbf{H}_{x \_ \text {_real }}, \mathbf{H}_{y \_ \text {_real }}$, and $\mathbf{H}_{z \_ \text {real }}$ contain the values of the measured magnetic field at all $N$ measurement points, while $\mathbf{H}_{x_{\text {_approx }}}, \mathbf{H}_{y \text { _approx }}$, and $\mathbf{H}_{z \_ \text {approx }}$ contain the values of the approximated magnetic field at the same locations.

\section{E. Converting to the Time Domain}

As the final goal of this method is to produce a time-domain approximation to a given electromagnetic noise source, it is imperative that the numerous optimized frequency-domain results produced over the required spectrum be combined into a single time-domain source. This time-domain source is obtained using a Fourier series of the individual frequency-domain solutions, as shown in following equation:

$$
I_{i}(t)=\sum_{\omega=1}^{n}\left|\mathbf{I}_{i}(\omega)\right| \sin \left(2 \pi f t+\angle \mathbf{I}_{i}(\omega)\right) .
$$

Here, $I_{i}(t)$ is the current in dipole $i$ at time $t$, and $\mathbf{I}_{i}(\omega)$ is the complex current phasor for the same dipole at frequency number $\omega$. The total number of frequencies used, $n$, is problem dependant. If using a linear range of frequencies, the gap between frequencies should be low enough so that all major components of the measured magnetic fields are accounted for. Typically, the gap between adjacent frequencies would be set between 1 and $10 \mathrm{MHz}$, or the minimum resolution of the spectrum analyzer used. Alternatively, a predefined set of frequencies can be used based on an initial investigation of the device under test.

\section{Case Study: Modeling Emissions FROM A TRANSMISSION LINE}

In order to demonstrate the source modeling method proposed in this paper, a simple transmission-line model is used. The model, shown in Fig. 3, consists of a transmission line with a right-angle bend. The shorter section of transmission line to the right is terminated to a $100-\mathrm{mm}$ square ground plane on the bottom layer of the printed circuit board (PCB) with a $1-\mathrm{k} \Omega$ resistive load. The $\mathrm{PCB}$ itself is $2 \mathrm{~mm}$ thick and has a relative permitivitty of 4.4. This transmission line is excited with a 50-MHz square-wave signal having rise and fall times of $1 \mathrm{~ns}$, as shown in Fig. 4.

\section{A. Generating the Approximate Model}

To generate the approximate model, the $x-, y$ - and $z$ components of the magnetic field generated by the transmission line were measured at $10 \mathrm{~mm}$ intervals in both the $x$ - and

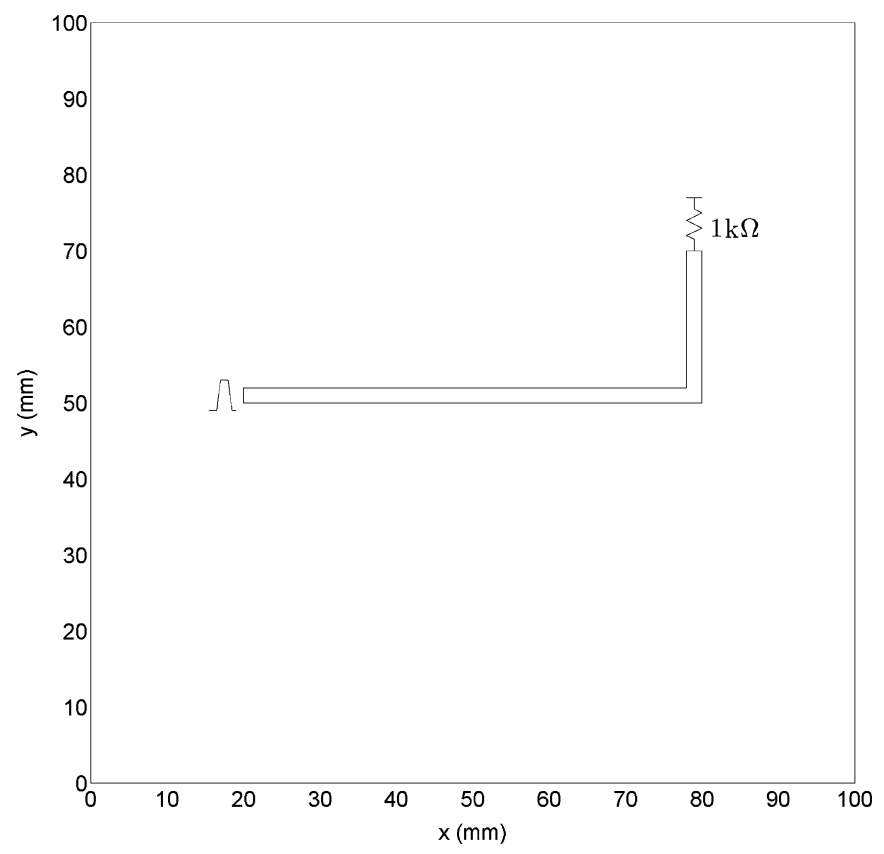

Fig. 3. Layout of the transmission-line case study. Note the ground plane on the under side is not shown.

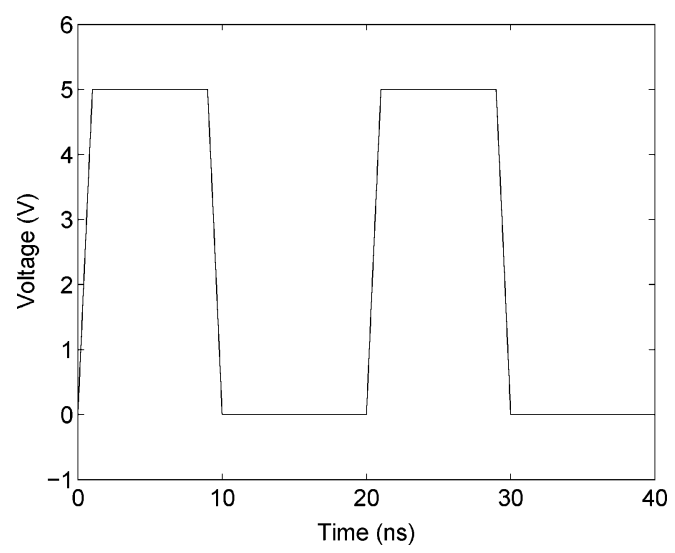

Fig. 4. Two periods of the transmission line model's square-wave excitation source.

$y$-directions starting from the origin $(0,0)$ in Fig. 3 . These measurements were performed at a fixed height of $20 \mathrm{~mm}$ above the PCB. A total of 242 dipoles were used, positioned, as shown in Fig. 5. Using the measurement data, an initial model was calculated over the frequency range $30 \mathrm{MHz}-1 \mathrm{GHz}$ at $10-\mathrm{MHz}$ intervals using (11) for the $x$-direction dipoles, and (12) for the $y$-direction dipoles. On a modern Intel processor running at $2.7 \mathrm{GHz}$, these calculations required less than a minute of computation time. This initial seed solution will henceforth be referred to as the unoptimized approximate model.

The final approximate model for this transmission-line test case was obtained by optimizing the seed solution at each frequency in the aforementioned range using the particle swarm algorithm described in Section III-C. This optimized model, which required about $30 \mathrm{~min}$ of computational time to derive, was then finally converted to a set of time-domain current sources. 


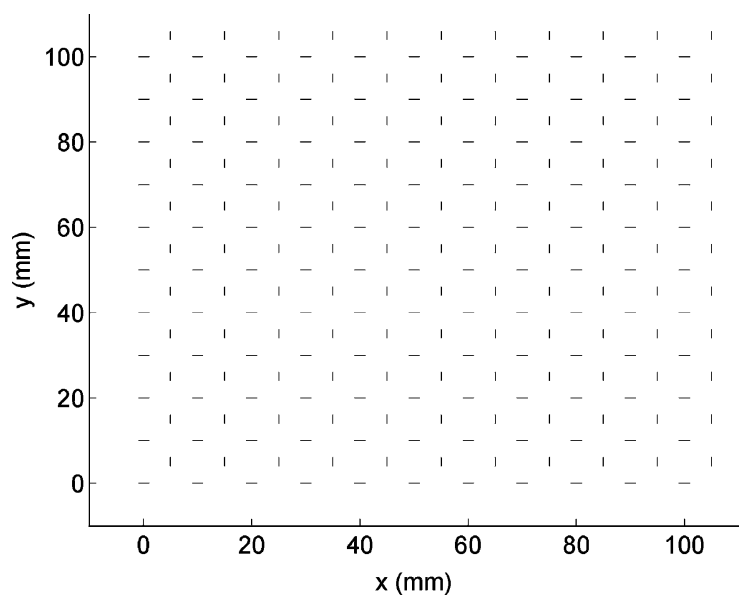

Fig. 5. Layout of dipoles in transmission-line approximate source model.

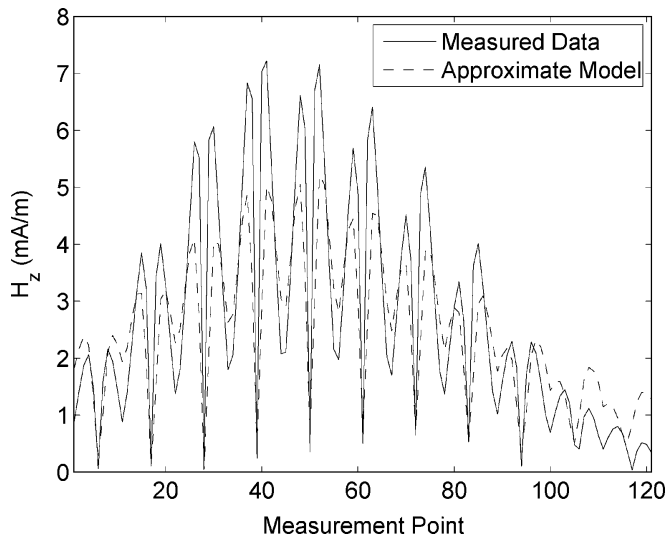

Fig. 6. Measured $H_{z}$ field strength $(f=100 \mathrm{MHz})$ at each measurement point compared to the equivalent unoptimized approximate field.

\section{B. Verification of the Model}

In order to verify that the procedure presented in this paper produces an optimized approximate model that is both an improvement over the original seed solution and an accurate representation of the noise source it is designed to model, two stages of verification and testing were performed. First, the magnetic fields produced by both the optimized and unoptimized approximate models at the measurement points were compared to the data obtained from measurement of the transmission-line device at the same points, $20 \mathrm{~mm}$ above the transmission-line PCB. Second, more physical measurements were made at a height of $40 \mathrm{~mm}$ above the transmission-line PCB, and these measurements compared to the results produced by the optimized approximate model at the same height.

Fig. 6 shows the $z$-component of the magnetic field at each measurement point produced by the unoptimized approximate model at a single selected frequency, $100 \mathrm{MHz}$, along with the measured data from the physical device. This frequency was selected as the average improvement at $100 \mathrm{MHz}$ was approximately $22 \%$, which is roughly the mean of the graph in Fig. 12, and therefore, this frequency point represents a typical case. The poor accuracy of the initial approximate model for this field component was expected, as the $z$-component of the measured field data was not used in the generation of this model.

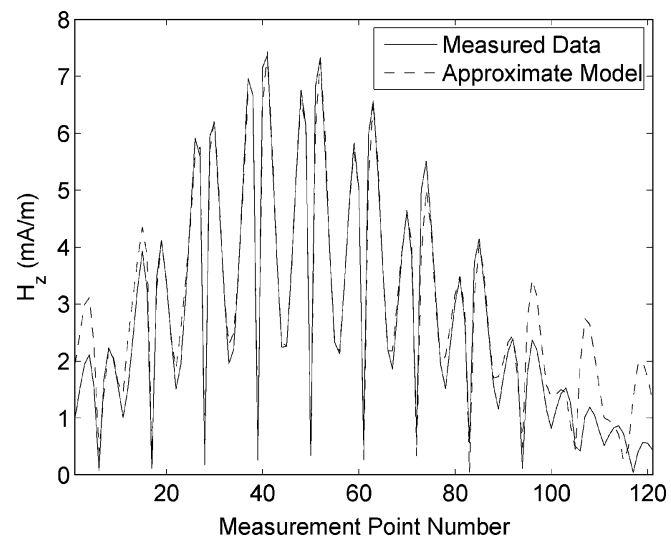

Fig. 7. Measured $H_{z}$ field strength $(f=100 \mathrm{MHz})$ at each measurement point compared to the equivalent optimized approximate field.

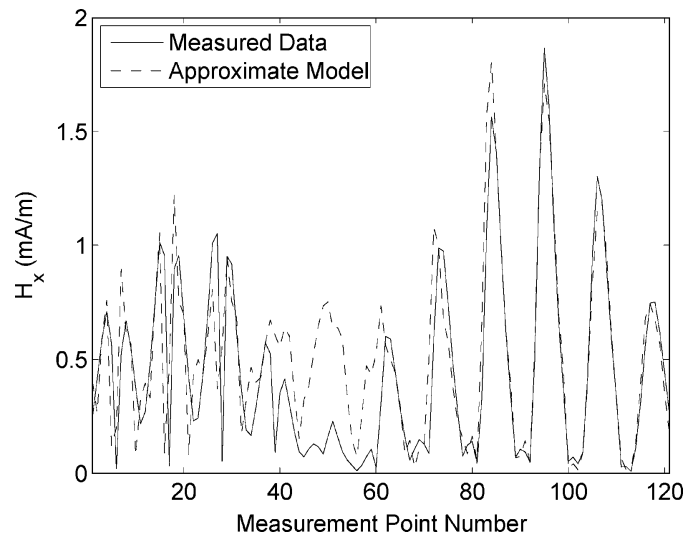

Fig. 8. Measured $H_{x}$ field strength ( $f=100 \mathrm{MHz}$ ) at each measurement point compared to the equivalent optimized approximate field.

When compared to the $z$-component of the magnetic field produced by the optimized approximate model at the same frequency and measurement points shown in Fig. 7, it can be seen that the optimization algorithm has significantly improved the accuracy of the approximate model in regards to $z$-component of the field. There is still some error present in the $z$-component of the optimized result around points 100-120, as the optimization algorithm focused on improving the accuracy of the approximate model in regions where the field strength is highest. Regions of high field strength are more important when assessing devices for compliance with EMC standards that are based on maximum allowable emissions. Significant improvement can be seen in these regions.

The original unoptimized approximate model was generated by ignoring the $z$-component of the magnetic field, and treating the $x$ - and $y$-components independently, which is possible due to the previously discussed restrictions placed on the orientation of the dipoles. Although Fig. 6 shows that this leads to significant error in the $z$-component of the magnetic field, it does allow this model to exactly replicate the fields produced by the device in question in the $x$ - and $y$-components of the magnetic field, at least at the points used to generate the model. As such, the $x$ - and $y$-components of the magnetic field produced by the optimized approximate model were examined, as shown in Figs. 8 and 9 respectively, to ensure that this accuracy has not 


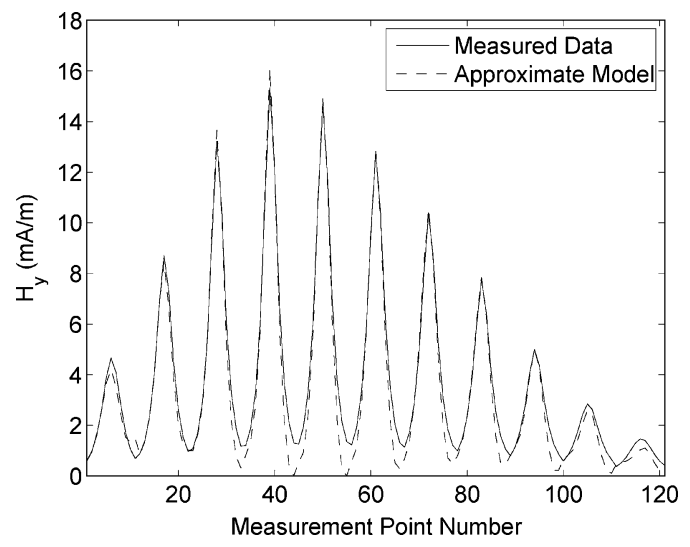

Fig. 9. Measured $H_{y}$ field strength $(f=100 \mathrm{MHz})$ at each measurement point compared to the equivalent optimized approximate field.

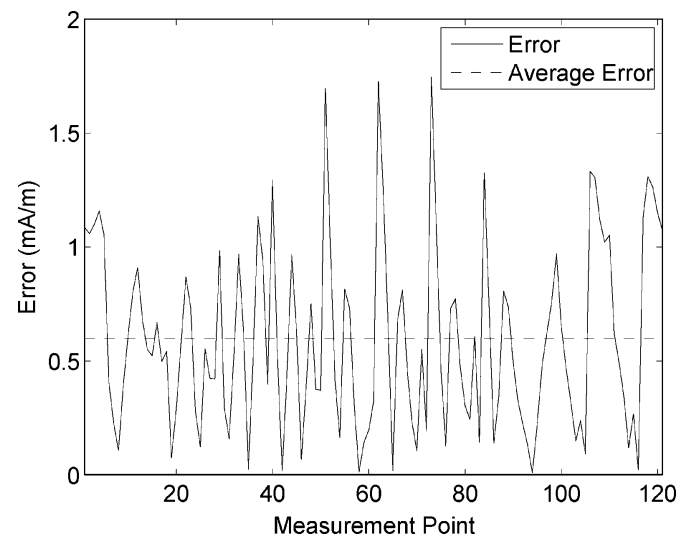

Fig. 10. Error in the $z$-component of the magnetic field generated by the unoptimized approximate model $(f=100 \mathrm{MHz})$.

been diminished as a results of improving the accuracy in terms of the $z$-component of the magnetic field.

From these results, it can be seen that the optimization algorithm results in very little error being introduced into the $y$-component of the magnetic field, although some error is introduced to the $x$-component, particularly around measurement point 50. However, both the maximum and average error in this data are significantly less than the error present in the $z$-component of the magnetic field produced by the unoptimized model, due to the greater magnitude of the $z$-component of the field.

To compare the optimized approximate model with the original unoptimized one, the errors between the magnetic fields produced by the approximate models and the measured data from the transmission-line device were calculated. Fig. 10 shows the average error in the $z$-component of the magnetic fields generated by the original unoptimized approximate model, and Fig. 11 shows the error measurements for the optimized approximate model.

Large errors can be seen at measurement points 52, 63, and 74 for the unoptimized approximate model. These points are located at $(x, y)$ points $(40 \mathrm{~mm}, 70 \mathrm{~mm}),(50 \mathrm{~mm}, 70 \mathrm{~mm})$, and (60 mm, $70 \mathrm{~mm}$ ), respectively (at the measurement height of $20 \mathrm{~mm}$ ). In relation to the transmission line shown in Fig. 3, these

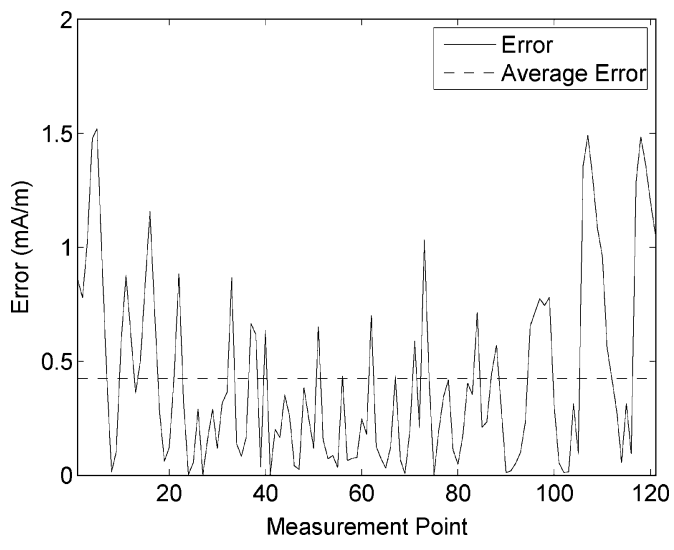

Fig. 11. Error in the $z$-component of the magnetic field generated by the optimized approximate model ( $f=100 \mathrm{MHz})$.

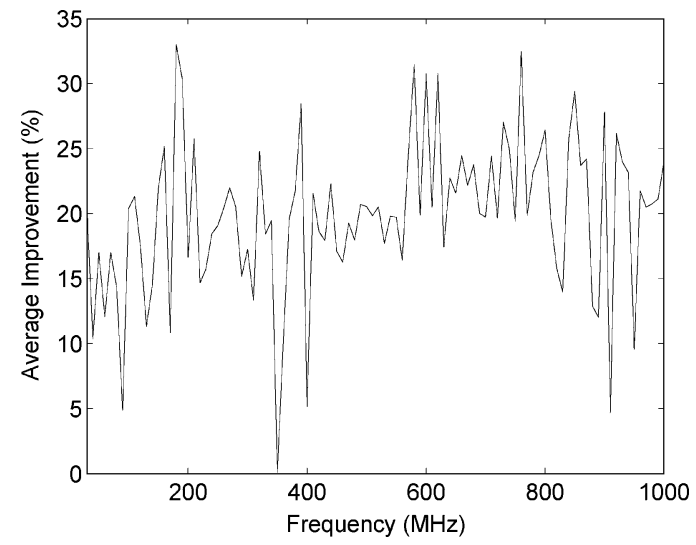

Fig. 12. Average improvement gained by optimization of the dipole currents.

points are located in an area where the $x$-oriented and $y$-oriented segments of transmission line will both be producing relatively strong $H_{z}$ fields. This is to be expected in this initial model, as it is produced by ignoring the $z$-component of the measured field. As such, it will place high currents on the $y$-direction dipoles near the $y$-oriented segment of transmission line (as this area will contain the highest $H_{x}$ fields), and high currents on the $x$ direction dipoles near the $x$-oriented segment (as this area will contain the highest $H_{y}$ fields), without considering the coupling in the $z$-component of the magnetic field. The optimization algorithm is able to remedy this shortfall, as seen in Fig. 11, as it does account for $z$-component of the magnetic field produced by the dipole array.

The average improvement obtained through the use of the optimization procedure can be defined as

$$
\text { average improvement }=\frac{1}{N} \sum_{i=1}^{N}\left(\frac{E_{u}-E_{o}}{E_{u}}\right)
$$

where $E_{u}$ is the average error present over all spatial components in the unoptimized approximate model, $E_{o}$ is the average error in the optimized model, and, as in previous equations, $N$ is the number of measurement points, in this case 121. As shown in Fig. 12, the optimized approximate model represents a significant improvement in accuracy over to the unoptimized 


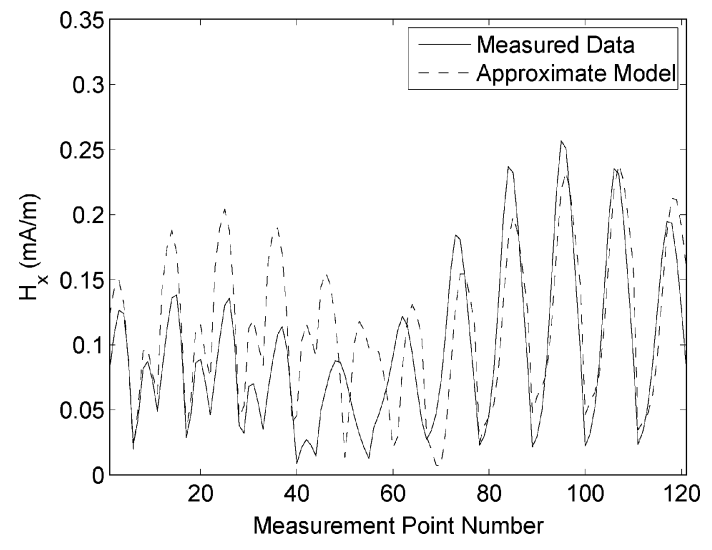

Fig. 13. Measured $H_{x}$ field strength compared to the equivalent $H_{x}$ field strength generated by the approximate model, $40 \mathrm{~mm}$ above the transmissionline PCB.

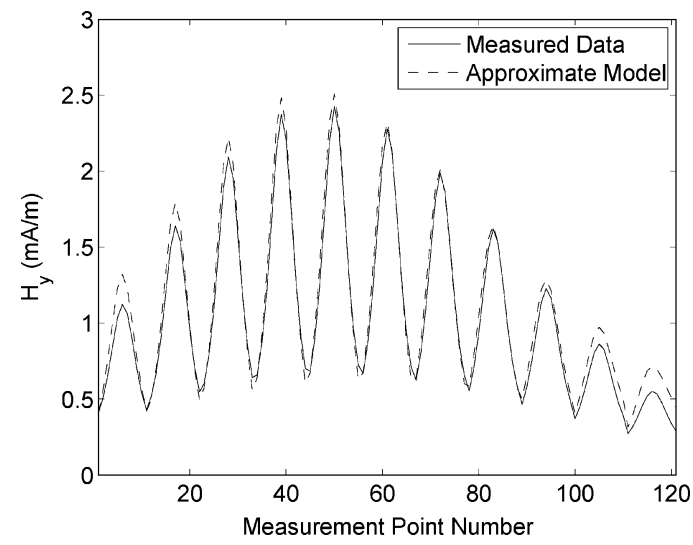

Fig. 14. Measured $H_{y}$ field strength compared to the equivalent $H_{y}$ field strength generated by the approximate model, $40 \mathrm{~mm}$ above the transmissionline PCB.

model, across the entire frequency range. It should be noted that while Figs. 10 and 11 demonstrate a 30\% improvement in $H_{z}$ at $100 \mathrm{MHz}$, the errors introduced in the $H_{x}$ and $H_{y}$ decreased the overall average improvement shown in Fig. 12 to approximately $22 \%$.

To verify that the approximate model generated is accurate at all heights above the device being modeled (in this case the transmission line), the magnetic fields produced by the device were again measured using the same grid of measurement points, this time at a height of $40 \mathrm{~mm}$ above the PCB. Figs. 1315 show the $H_{x}, H_{y}$, and $H_{z}$ components (respectively) of the measured magnetic field as well as the magnetic fields produced by the optimized approximate model at the same measurement points.

From these figures, it can be seen that the optimized approximate model generated using the procedure presented in this paper produces accurate magnetic fields at multiple heights above the device being modeled, verifying the accuracy and usefulness of the model for modeling near-field radiated emissions from an arbitrary device. In order to demonstrate the accuracy of the method in the far-field, far-field radiation patterns at a frequency of $100 \mathrm{MHz}$ were calculated through FDTD modeling of both

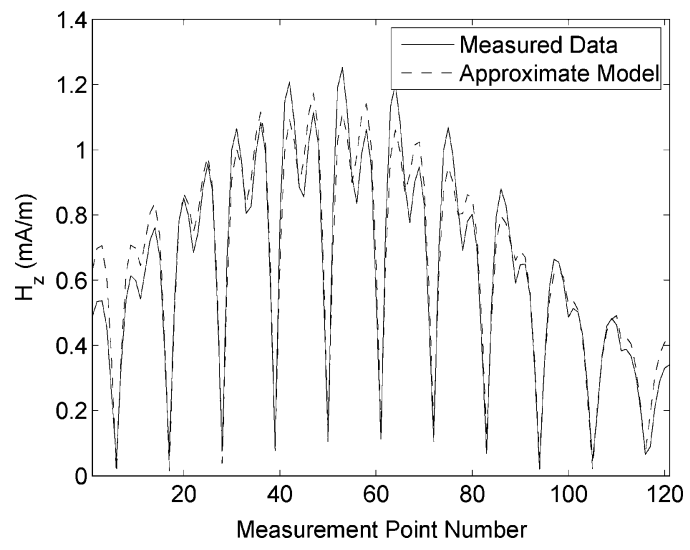

Fig. 15. Measured $H_{z}$ field strength compared to the equivalent $H_{z}$ field strength generated by the approximate model, $40 \mathrm{~mm}$ above the transmissionline PCB.

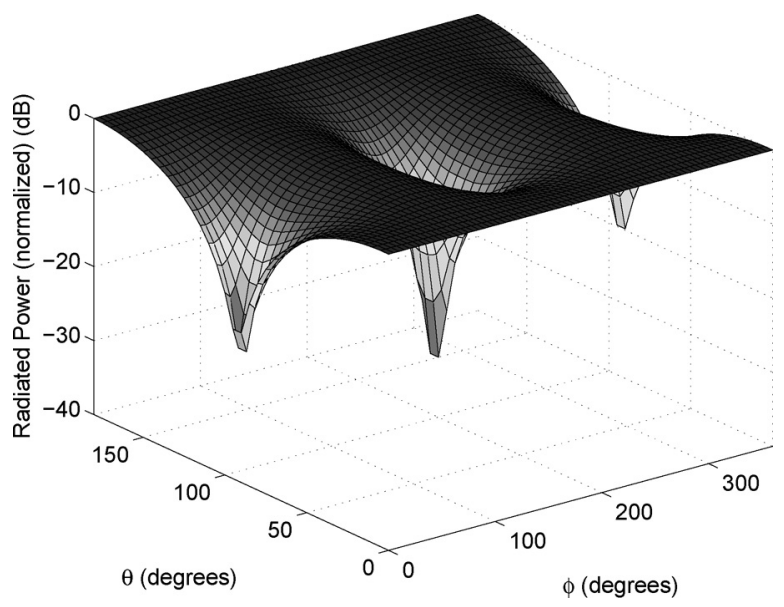

Fig. 16. Far-field radiation generated by the transmission-line PCB device.

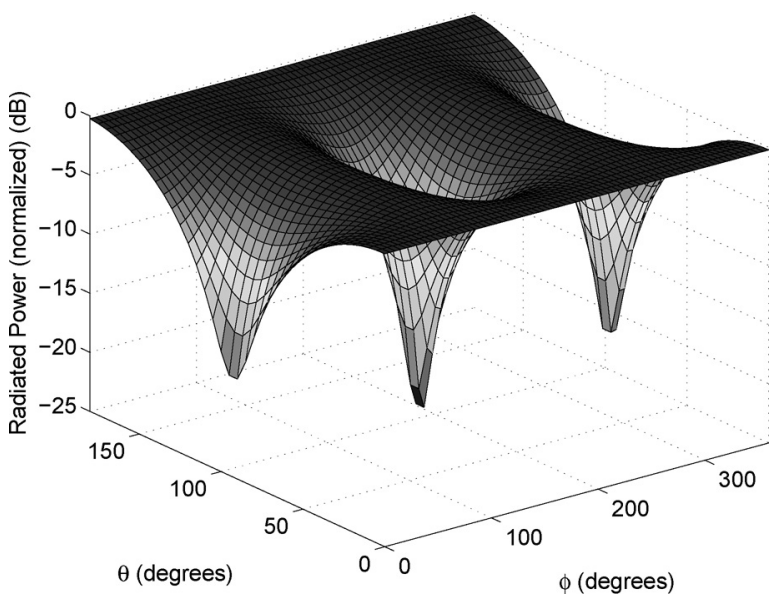

Fig. 17. Far-field radiation generated by the approximate model.

the actual transmission-line model and the approximate dipole array, as shown in Figs. 16 and 17.

In these figures, the $\phi$ and $\theta$ axes represent the azimuth and elevation of the far-field measurement point, as shown in Fig. 18. The radiation pattern produced by the transmission-line device resembles that produced by an electric dipole antenna oriented 


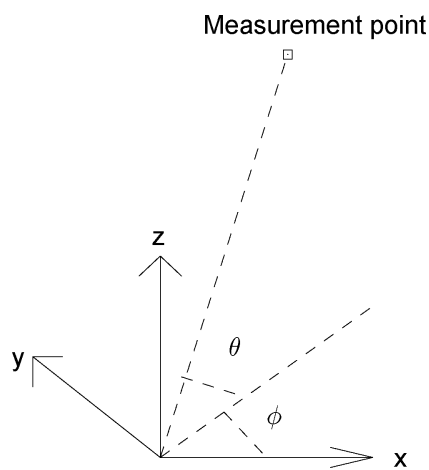

Fig. 18. Spherical coordinate system used for the far-field radiation patterns.

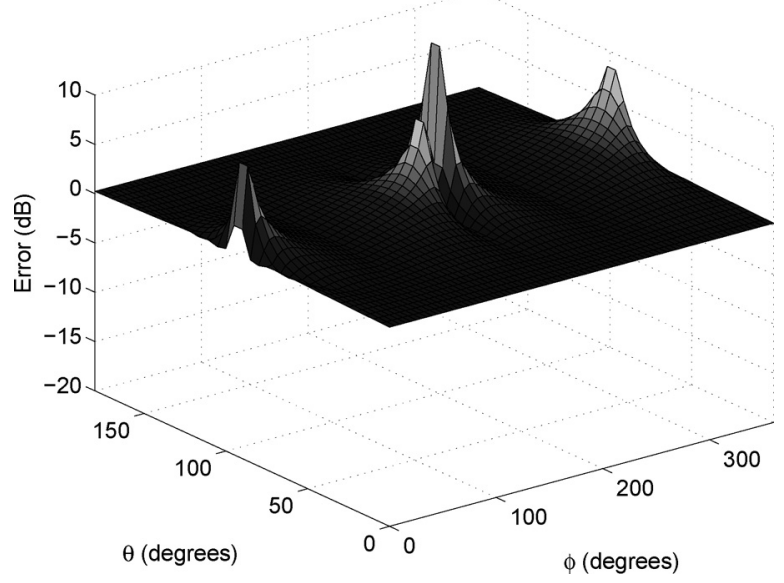

Fig. 19. Difference between far-field radiation patterns produced by the transmission-line device and the approximate model.

along the $x$-axis due to the longer transmission-line segment, with a slight rotation caused by the shorter segment. It can be seen that the approximate source model produces a highly accurate representation of the transmission line's far-field radiation pattern, with the $0.4-\mathrm{dB}$ error at peak of the radiation pattern not discernable from the figures. The larger errors seen in Fig. 19 are caused by a slight difference in rotation between the two radiation patterns.

\section{CONCLUSION}

The EM noise source approximation method presented in this paper has been shown to be capable of producing very accurate approximate models for use in FDTD simulations. The method uses an array of electric dipoles which are restricted to either the $x$ - or $y$-orientation by the FDTD grid. An initial model based on a direct solution of the equations governing radiation from electric dipole antennas and measured $H_{x}$ and $H_{y}$ data were not sufficiently accurate in regard to the $z$-component of the magnetic field being modeled. Through the use of a PSO algorithm, the current phasors exciting the dipoles are optimized to minimize the overall error present in the approximation.

This source modeling method was applied to a transmissionline device in order to verify its ability to produce accurate results. Using $121 x$-direction dipoles and $121 y$-direction dipoles, the method was able to produce an approximate model that closely matched the near-field radiated fields from the transmission-line PCB not only at the positions used to generate the model, but also at a distance twice as far again from the PCB. Furthermore, the approximate model generated using the proposed method was able to replicate the far-field radiation produced by the transmission-line device to a high degree of accuracy.

\section{REFERENCES}

[1] A. Dolente, U. Reggiani, and L. Sandrolini, "Comparison of radiated emissions from different heatsink configurations," in Proc. IEEE 6th Int. Symp. Electromagn. Compat. Electromagn. Ecol., 2005, pp. 49-53.

[2] J. Lu and X. Duan, "EMC computer modelling techniques for CPU heat sink simulation," in Proc. 3rd Int. Conf. Comput. Electromagn. Appl., 2004, pp. 272-275.

[3] J. Lu and F. Dawson, "EMC computer modelling techniques for CPU heat sink simulation,” IEEE Trans. Magn., vol. 42, no. 10, pp. 3171-3173, Oct. 2006.

[4] C. Wang, J. L. Drewniak, J. L. Knighten, D. Wang, R. Alexander, and D. M. Hockanson, "Grounding of heatpipe/heatspreader and heatsink structures for EMI mitigation," in Proc. IEEE Symp. Electromagn. Compat., 2001, vol. 2, pp. 916-920.

[5] L. Covert and J. Lin, "Simulation and measurement of a heatsink antenna: A dual-function structure," IEEE Trans. Antennas Propag., vol. 54, no. 4, pp. 1342-1345, Apr. 2006.

[6] Y. Vives-Gilabert, C. Arcambal, A. Louis, F. de Daran, P. Eudeline, and B. Mazari, "Modelling magnetic radiations of electronic circuits using near-field scanning method," IEEE Trans. Electromagn. Compat., vol. 49, no. 2, pp. 391-400, May 2007.

[7] J-R. Regué, M. Ribó, J.-M. Garrell, and A. Martín, “A genetic algorithm based method for source identification and far-field radiated emissions prediction from near-field measurements for PCB characterization," IEEE Trans. Electromagn. Compat., vol. 43, no. 4, pp. 520-530, Nov. 2001.

[8] K. Yee, "Numerical solution of initial boundary value problems involving Maxwell's equations in isotropic media," IEEE Trans. Antennas Propag., vol. AP-14, no. 3, pp. 302-307, May 1966.

[9] J. Kennedy and R. Eberhart, "Particle swarm optimization," in Proc. IEEE Int. Conf. Neural Netw., 1995, pp. 1942-1948.

[10] A. P. Engelbrecht, Fundamentals of Computational Swarm Intelligence. Chichester, U.K.: Wiley, 2005.

[11] C.-H. Chou and J.-N. Chen, "Genetic algorithms: Initialization schemes and genes extraction," in Proc. 9th IEEE Int. Conf. Fuzzy Syst., 2000, vol. 2, pp. 965-968.

[12] S. Rahnamayan, H. R. Tizhoosh, and M. M. A. Salama, "A novel population initialization method for accelerating evolutionary algorithms," Comput. Math. Appl., vol. 53, no. 10, pp. 1605-1614, 2007.

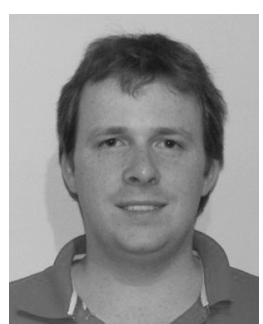

Ian Scriven (S'07-M'09) was born in Ipswich, Qld., Australia, in 1984. He received the electrical engineering and computer science degrees in 2006 from Griffith University, Brisbane, Qld., where he is currently working toward the Ph.D. degree in the same areas.

His current research interests include computational electromagnetics, EMC, and decentralized particle swarm optimization algorithms. 


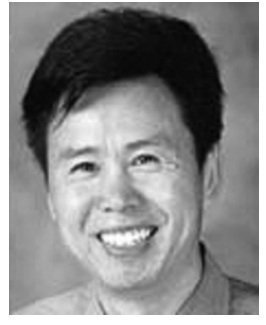

Junwei Lu (M'92-SM'05) received the B.Eng. degree in electrical engineering from Xian Jiaotong University, Xian, China, in 1976, the M.Eng. degree in electronic and computer engineering from the National Toyama University, Toyama, Japan, in 1988, and the Ph.D. degree in electrical and computer engineering from the National Kanazawa University, Kanazawa, Japan, in 1991.

From 1976 to 1984 , he was with the Institute of Qinhai Electric Power Testing and Research, China, where he was involved in the various national research projects for the electrical power industry. During 1985, he was with the Laboratory of Electrical Communications, Toyama University, Japan, where he was involved in the area of computational electromagnetics. During 1988, he was with the Laboratory of Electrical Energy Conversion, Kanazawa University, where he was involved in the applied computational electromagnetics and was involved in the development of magnetics devices. In 1992, he joined the School of Microelectronic Engineering, Griffith University, Brisbane, Qld., Australia, where he is currently an Associate Professor. His research interests include computational and visual electromagnetics, EMC computer modeling and simulation, smart mobile terminal antennas and RF/MW devices and circuits, and high-frequency magnetics and power electronics.

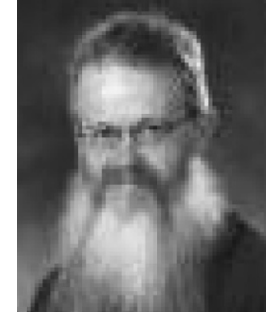

Andrew Lewis received the B.Eng. degree in computer engineering from the University of Newcastle, Tyne and Wear, U.K., in 1984 and the Ph.D. degree in computer science from Griffith University, Qld., Australia, in 2004.

$\mathrm{He}$ is a Senior Research Specialist in Research Computing Services, Griffith University, Brisbane, Qld., Australia, where he is an Adjunct Senior Lecturer with ICT. He was with BHP Billiton, where he was engaged in industrial applied research. His current research interests include parallel optimization algorithms for large numerical simulations, including gradient descent, direct search methods, evolutionary programming, particle swarm and ant colony systems, multiobjective optimization techniques for engineering design, and parallel, distributed, and grid computing methods. He has authored or coauthored in the area of optimization algorithms and applications. 